\title{
Evaluation of the Liver Disease Information in Baidu Encyclopedia and Wikipedia: Longitudinal Study
}

Fei Sun, MD; Fuchun Yang, MD; Shusen Zheng, MD, PhD

Division of Hepatobiliary and Pancreatic Surgery, Department of Surgery, The First Affiliated Hospital, Zhejiang University School of Medicine, Hangzhou, China

Corresponding Author:

Shusen Zheng, MD, PhD

Division of Hepatobiliary and Pancreatic Surgery, Department of Surgery

The First Affiliated Hospital

Zhejiang University School of Medicine

\#79 Qingchun Road

Hangzhou, 310003

China

Phone: 8613805749805

Email: shusenzheng@zju.edu.cn

\section{Abstract}

Background: The internet has changed the way of people acquiring health information. Previous studies have shown that Wikipedia is a reasonably reliable medical resource, and it has been ranked higher than other general websites in various search engines. Baidu Encyclopedia is one of the most popular encyclopedia websites in China. However, no studies have shown the quality of the content provided in the Baidu Encyclopedia.

Objective: This study aimed to evaluate the quality of liver disease information provided by Wikipedia (in English) and Baidu Encyclopedia (in Chinese) and to perform a comparison of the quality and timeliness of the articles published in these two encyclopedias. Moreover, a 3-year follow-up study was conducted to compare if the information in both these websites was updated regularly over this period.

Methods: We searched for information on liver diseases by using the International Statistical Classification of Diseases and Related Health Problems 10th Revision Version 2016 codes on Wikipedia (in English) and Baidu Encyclopedia (in Chinese). The quality of the articles was assessed using the DISCERN instrument, which consists of 3 sections. We recorded the latest editing date of the webpages and calculated the date interval to evaluate the update timeliness of these websites.

Results: We found 22 entries on liver diseases in Baidu Encyclopedia and 15 articles in Wikipedia between September 15, 2016, and September 30, 2016, and we found 25 entries in Baidu Encyclopedia and 16 articles in Wikipedia between September 15, 2019, and September 30, 2019. In section 1 of the DISCERN instrument, the mean (SE) scores of Baidu Encyclopedia entries were significantly lower than those of Wikipedia articles. In section 2 and section 3 of the DISCERN instrument, the DISCERN scores of Baidu Encyclopedia entries were lower than those of Wikipedia articles, but the differences were not statistically significant. The total DISCERN scores of Baidu Encyclopedia entries were significantly lower than those of Wikipedia articles. The update interval of the entries in Baidu Encyclopedia was found to be significantly longer than that of the articles in Wikipedia.

Conclusions: This study shows that the quality of articles and the reliability of the research content on liver diseases in Wikipedia are better than those of the entries in Baidu Encyclopedia. However, the quality of the treatment choices provided in both Wikipedia and Baidu Encyclopedia is not satisfactory. Wikipedia is updated more frequently than Baidu Encyclopedia, thereby ensuring that the information presented has the most recent research findings. The findings of our study suggest that in order to find accurate health information, it is important to seek the help of medical professionals instead of looking for a prescription amid the confusing information provided on the internet.

(J Med Internet Res 2021;23(1):e17680) doi: $\underline{10.2196 / 17680}$

\section{KEYWORDS}

Baidu Encyclopedia; Wikipedia; internet; website; liver disease; health information; DISCERN instrument; timeliness 


\section{Introduction}

Over 4.5 billion internet users were reported worldwide in June 2019, with 854 million of them being in China [1,2]. Nowadays, the internet has become the primary source of information, and it has changed the way of people acquiring medical and health information. More than $50 \%$ of the internet users in the United States of America have been reported to search for web-based health care-related information $[3,4]$. A study in China found that $87.8 \%$ of the patients with scoliosis searched for scoliosis-related information on the internet, thereby indicating the high proportion of internet usage as a source of health information [5]. Increasing number of patients are seeking information about their diseases on the internet, but the reliability of web-based health care-related information is still questionable [6,7].

Wikipedia is a web-based encyclopedia that provides valuable web-based health information; it contains more than 5,956,750 articles in English [8-10]. Previous studies have shown that Wikipedia is a reasonably reliable medical resource and it was ranked higher than other general websites in search engines $[8,11]$, although there are some errors in Wikipedia articles compared to peer-reviewed sources [12]. Baidu Encyclopedia, Wikipedia's equivalent in China, contains more than 16,244,000 entries in Chinese [13]. Wikipedia and Baidu Encyclopedia are the most popular and consulted encyclopedia websites in English and Chinese, respectively [14-16]. On December 9, 2012, Baidu Encyclopedia announced the "rainbow plan," wherein all medical entries could only be edited and revised by certified medical experts, which would improve the quality of the health information provided in Baidu Encyclopedia [17]. However, no study has yet examined the quality of the data provided in Baidu Encyclopedia.

Liver disease is among the top 10 causes of death in middle-income and high-income countries [18]. We have been engaged in hepatic surgery for many years and we are competent in the diagnosis and treatment of liver diseases. Therefore, we selected liver diseases as the object of analysis in this study. This study aimed to evaluate the quality of liver disease information provided by Wikipedia in English and Baidu Encyclopedia in Chinese and we aimed to perform a comparison of the quality of the research in these resources and the timeliness of the recent updates in the research between these 2 resources. Since the update frequency on the internet is high by day and by hour, 3 years can be considered as an extended period for evaluating the changes in the information over a long interval during follow-up. Thus, 3-year monitoring was conducted to compare whether the information on these websites was improved over this period. Our research results will help readers judge the reliability of web-based encyclopedia entries and avoid the medical problems caused by believing unreliable materials on the internet.

\section{Methods}

\section{Data Sources}

The articles analyzed in this study are available in Wikipedia [9] in English and in Baidu Encyclopedia [13] in Chinese. Data were compiled between September 15, 2016, and September 30, 2016. For the 3-year follow-up, data were compiled between September 15, 2019, and September 30, 2019. We chose 3 years for the length of the comparison because 3 years is a widely used and acceptable follow-up time [19-21].

\section{Retrieval of Liver Disease Articles}

The selection of topics was based on the International Statistical Classification of Diseases and Related Health Problems 10th Revision (ICD-10) version 2016. ICD defines almost all of the health-related conditions, and it is the diagnostic classification standard for all clinical and research purposes. In the ICD-10 version 2016, diseases of the liver are classified under Chapter XI Diseases of the digestive system, with categories K70 to K77 [22]. The Baidu Encyclopedia (in Chinese) and Wikipedia (in English) were investigated for articles on ICD-10 version 2016 codes. All ICD-10 code titles on the diseases of the liver were used for the search. Entries without search results were excluded from the study. Search terms on Baidu Encyclopedia (in Chinese) and Wikipedia (in English) are listed in the Multimedia Appendices 1 and 2, respectively. The proportion of the available search results were recorded and analyzed.

\section{Assessment of the Quality of the Research Articles}

The quality of the articles was assessed using the DISCERN instrument, which is used for judging the quality of health information on treatment choices [23]. The use of the DISCERN instrument for information in encyclopedias is controversial. Although some studies believe that the original edition of the DISCERN instrument is not suitable for evaluating Wikipedia articles [24,25], there are still many recent studies that have used the DISCERN instrument to evaluate the quality of Wikipedia articles $[15,16]$. Thus, the DISCERN instrument can be considered as an appropriate tool for evaluating the quality of Wikipedia articles in the absence of a better instrument.

The DISCERN instrument consists of 16 questions that are rated on a 5-point scale (1=definitely no, 5=definitely yes) (Multimedia Appendix 3). All these 16 questions are categorized into 3 sections. Section 1 (questions 1 to 8 ) assesses the reliability of a paper, section 2 (questions 9 to 15) focuses on the quality of the treatment information, and section 3 (question 16) evaluates the overall quality [23]. A higher DISCERN score indicates better paper quality. The overall DISCERN score ranges from 16 to 80 and the articles were categorized as very poor (16-26), poor (27-38), fair (39-50), good (51-62), and excellent (63-80) based on the scores $[15,16]$.

All 3 authors have been engaged in hepatobiliary and pancreatic surgery for many years and are competent in the diagnosis and treatment of liver diseases. We have mastered the professional knowledge of liver diseases and can make professional evaluations. Sun and Yang used the DISCERN instrument to evaluate the articles in Baidu Encyclopedia (in Chinese) and Wikipedia (in English), respectively. The DISCERN score was determined through discussions. If no agreement was reached during the debate, Zheng was consulted to make the final judgment. Subsequently, Sun and Yang agreed on all the ratings. 


\section{Timeliness of the Updated Articles}

We recorded and analyzed the last editing date of the web page. In 2016, the update interval was calculated as the date interval between the updated date and September 20, 2016. In 2019, the update interval was calculated as the date interval between the updated date and September 20, 2019. The update interval reflects the update frequency of a webpage. We compared the update interval of each paper to evaluate the update timeliness of the website.

\section{Statistical Analysis}

For the statistical analysis, means and standard errors (mean [SE]) were calculated. $P$ values less than .05 were considered significant. Differences between groups were assessed using two-tailed Student $t$ test with Welch correction. Statistical analyses were performed using GraphPad Prism 6 software (GraphPad Prism Software Inc).

\section{Results}

\section{Retrieval of Liver Disease Articles}

We searched Baidu Encyclopedia and Wikipedia with the entries in the ICD-10 diseases of the liver category. A total of 8 liver disease categories were classified, with categories K70 to K77. Only a portion of the entries were retrieved as articles in the search results. We found 22 entries in Baidu Encyclopedia and 15 articles in Wikipedia in 2016 and 25 entries in Baidu Encyclopedia and 16 articles in Wikipedia in 2019 (Table 1). In general, we were able to retrieve more entries in Baidu Encyclopedia than in Wikipedia.

Table 1. The proportion of the available search results in Baidu Encyclopedia and Wikipedia in 2016 and 2019.

\begin{tabular}{|c|c|c|c|c|c|}
\hline ICD- $10^{\mathrm{a}}$ category & $\begin{array}{l}\text { Entries included in the } \\
\text { ICD-10 classification } \\
(n=50)\end{array}$ & $\begin{array}{l}\text { Baidu Encyclopedia } \\
\text { entries in } 2016(n=22)\end{array}$ & $\begin{array}{l}\text { Wikipedia articles } \\
\text { in } 2016(n=15)\end{array}$ & $\begin{array}{l}\text { Baidu Encyclopedia } \\
\text { entries in } 2019(n=25)\end{array}$ & $\begin{array}{l}\text { Wikipedia articles } \\
\text { in } 2019(n=16)\end{array}$ \\
\hline K70 Alcoholic liver disease & 6 & 4 & 3 & 4 & 3 \\
\hline K71 Toxic liver disease & 10 & 1 & 1 & 1 & 1 \\
\hline $\begin{array}{l}\text { K72 Hepatic failure, not else- } \\
\text { where classified }\end{array}$ & 3 & 1 & 1 & 2 & 1 \\
\hline $\begin{array}{l}\text { K73 Chronic hepatitis, not } \\
\text { elsewhere classified }\end{array}$ & 5 & 3 & 1 & 3 & 1 \\
\hline $\begin{array}{l}\text { K74 Fibrosis and cirrhosis of } \\
\text { liver }\end{array}$ & 7 & 4 & 1 & 5 & 1 \\
\hline $\begin{array}{l}\text { K75 Other inflammatory liver } \\
\text { diseases }\end{array}$ & 7 & 3 & 2 & 3 & 2 \\
\hline K76 Other diseases of liver & 10 & 6 & 6 & 7 & 7 \\
\hline $\begin{array}{l}\text { K77 Liver disorders in diseases } \\
\text { classified elsewhere }\end{array}$ & 2 & 0 & 0 & 0 & 0 \\
\hline
\end{tabular}

${ }^{a}$ ICD-10: International Statistical Classification of Diseases and Related Health Problems 10th Revision.

\section{Assessment of the Quality of Articles}

We used the DISCERN instrument to score the articles retrieved in Baidu Encyclopedia and Wikipedia and compared the section 1, section 2, section 3, and total scores (Figure 1). The results of the DISCERN instrument were comparable in 2016 and 2019. In section 1, the mean (SE) DISCERN score of Baidu Encyclopedia entries was significantly lower than that of Wikipedia articles (18.00 [SE 1.040] vs 26.60 [SE 1.359], $P<.001$ in 2016, respectively; 19.00 [SE 0.9110] vs 27.88 [SE 0.8892 ], $P<.001$ in 2019, respectively). In section 2 and section 3 of the DISCERN instrument, the mean DISCERN scores of Baidu Encyclopedia entries were lower than those of Wikipedia articles, but the differences were not statistically significant. Overall, the total scores of Baidu Encyclopedia entries were significantly lower than those of Wikipedia articles (33.68 [SE 2.265] vs 44.67 [SE 3.172], $P=.009$ in 2016, respectively; 34.72
[SE 1.943] vs 46.44 [SE 2.669], $P=.001$ in 2019, respectively) (Table 2).

The total DISCERN scores were categorized. In Baidu Encyclopedia in 2016, 9 entries were found to be of fair quality, 8 were of poor quality, and 8 were of very poor quality. In 2019, 10 entries were of fair quality, 11 were of poor quality, and 4 were of very poor quality. In Wikipedia in 2016, 5 articles were of good quality, 5 were of fair quality, 3 were of poor quality, and 2 were found to be of very poor quality. In 2019, 6 articles were found to be of good quality, 4 were of fair quality, and 5 were of poor quality.

Over time, the number of entries in Baidu Encyclopedia and Wikipedia showed an increase (Table 1). However, no significant differences were found on comparing the changes in the DISCERN scores of Baidu and Wikipedia entries over 3 years. 
Figure 1. The DISCERN scores of Baidu Encyclopedia entries and Wikipedia articles on liver diseases in 2016 and 2019 . $* * P<.01$, **** $P<.001$. BE-2016: Baidu Encyclopedia entries in 2016; BE-2019: Baidu Encyclopedia entries in 2019; W-2016: Wikipedia articles in 2016; W-2019: Wikipedia articles in 2019; ns: no significant difference.

Section 1
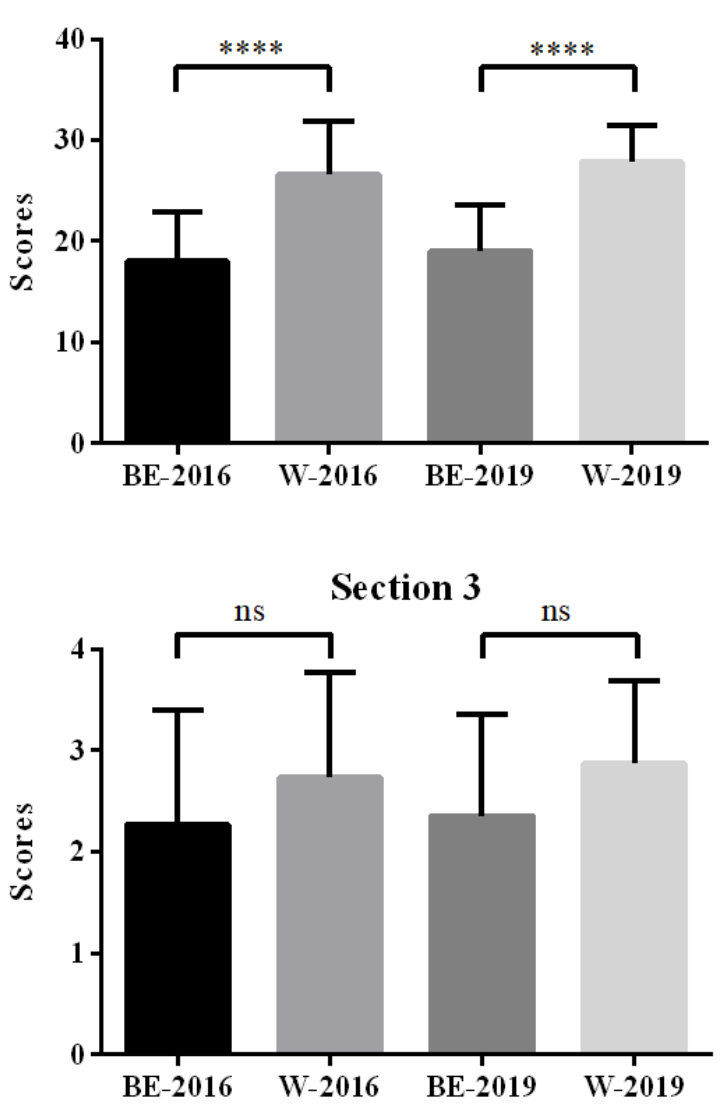
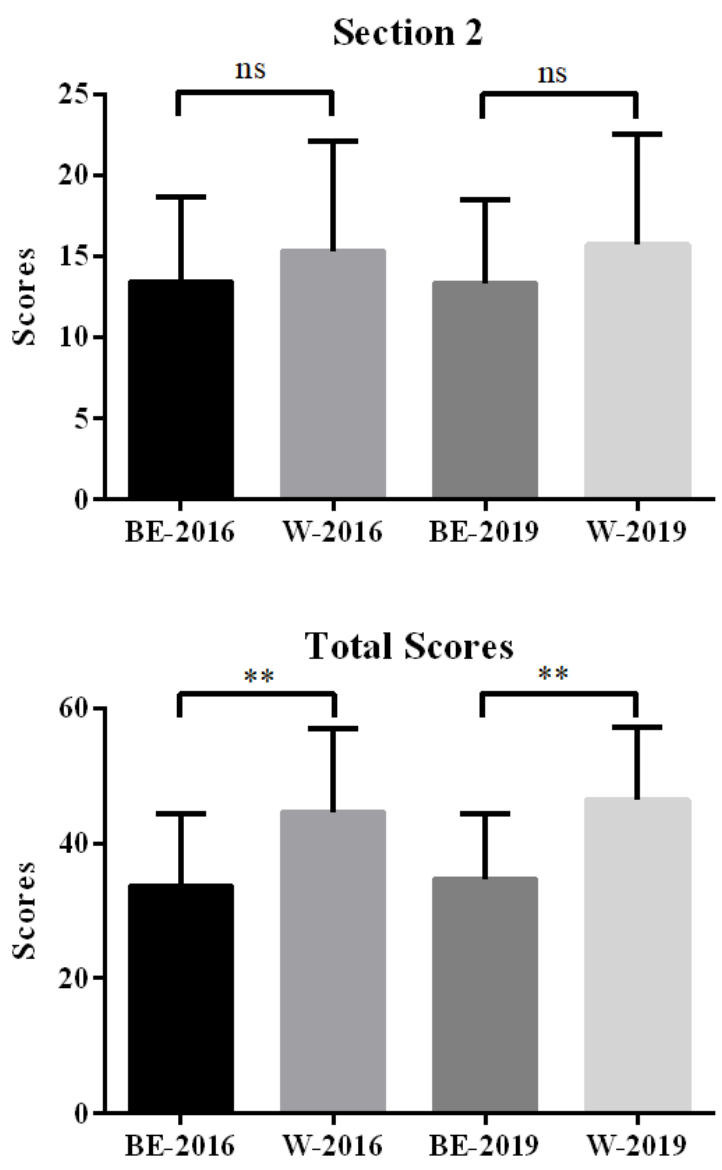

Table 2. The DISCERN scores of Baidu Encyclopedia and Wikipedia articles on liver diseases.

\begin{tabular}{|c|c|c|c|}
\hline Year, sections of DISCERN & Baidu Encyclopedia, mean (SE) & Wikipedia, mean (SE) & $P$ value \\
\hline \multicolumn{4}{|l|}{2016} \\
\hline Section 1 & $18.00(1.040)$ & $26.60(1.359)$ & $<.001$ \\
\hline Section 2 & $13.36(1.023)$ & $15.69(1.717)$ & .25 \\
\hline Section 3 & $2.273(0.2389)$ & $2.733(0.2667)$ & .21 \\
\hline Total & $33.68(2.265)$ & $44.67(3.172)$ & .009 \\
\hline \multicolumn{4}{|l|}{2019} \\
\hline Section 1 & $19.00(0.9110)$ & $27.88(0.8892)$ & $<.001$ \\
\hline Section 2 & $13.36(1.023)$ & $15.69(1.717)$ & .25 \\
\hline Section 3 & $2.360(0.1990)$ & $2.875(0.2016)$ & .07 \\
\hline Total & $34.72(1.943)$ & $46.44(2.669)$ & .001 \\
\hline
\end{tabular}

\section{Timeliness of Articles}

The update interval represents the timeliness of the updates on the website. We compared the update interval between Baidu Encyclopedia and Wikipedia (Figure 2). In 2016, the mean (SE) update interval of Baidu Encyclopedia entries was 571.6 (74.96) days and the mean (SE) update interval of Wikipedia entries was 55.93 (11.92) days. The update interval for entries in Baidu
Encyclopedia was significantly longer than that for articles in Wikipedia $(P<.001)$. After 3 years of follow-up, it was found that 11 of the 25 entries in Baidu Encyclopedia were recently edited before September 20, 2016, which meant that they had not been updated in the last 3 years and were in a state of loss of maintenance. In 2019, the mean (SE) update interval of Baidu Encyclopedia entries was 1067 (131.2) days and the mean (SE) 
update interval of Wikipedia articles was 27.44 (11.98) days. The update interval of the entries in Baidu Encyclopedia is significantly longer than that of the articles in Wikipedia
$(P<.001)$ (Figure 2). Therefore, the update timeliness of the articles in Wikipedia in 2016 and 2019 is significantly better than that of the entries in Baidu Encyclopedia.

Figure 2. The update interval of Baidu Encyclopedia entries and Wikipedia articles on liver diseases in 2016 and 2019. ** $P<.01$, **** $P<.001$. BE-2016: Baidu Encyclopedia entries in 2016; BE-2019: Baidu Encyclopedia entries in 2019; W-2016: Wikipedia articles in 2016; W-2019: Wikipedia articles in 2019; ns: no significant difference.

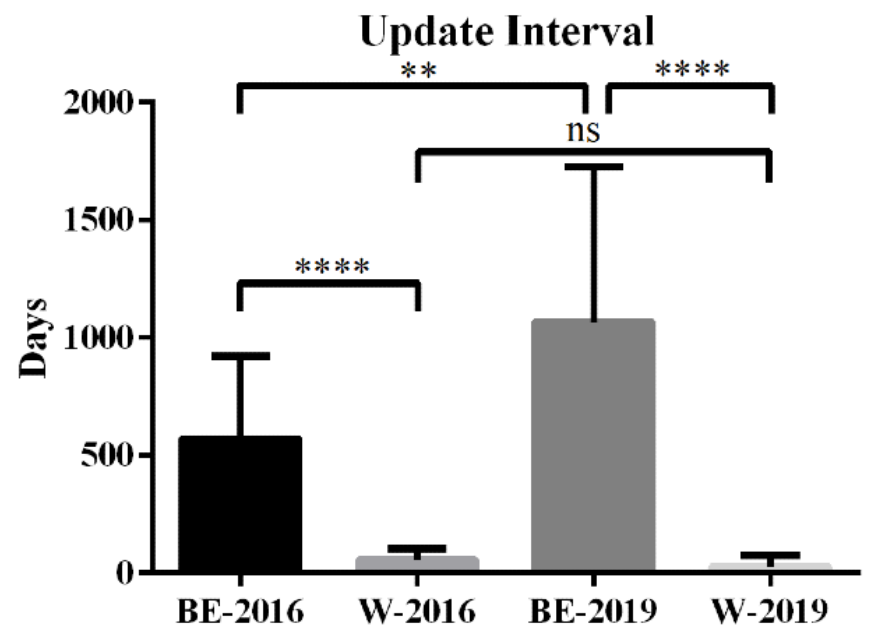

\section{Discussion}

\section{Principal Findings}

The main findings of this study were that the reliability of the articles on Wikipedia is better than that of the entries in Baidu Encyclopedia. However, the quality of the treatment information in both the encyclopedia websites is not satisfactory. With the increasing popularity of the internet, increasing number of patients are seeking health information and even treatment plans for their diseases on the internet [4]. However, the quality of the medical information on the internet is uneven, and a large proportion of the information is unreliable [6]. Previous research has shown that health information in a search engine is less reliable [26-28]. Wikipedia is a remarkable source of web-based health information [8]. Therefore, we analyzed the information on liver diseases in Baidu Encyclopedia in Chinese and Wikipedia in English to compare the quality of the medical information in these encyclopedia websites. The quality of the health information on the internet was studied over a 3-year follow-up period to see if it improved over time.

We evaluated the articles on liver diseases in Baidu Encyclopedia and Wikipedia in terms of quantity and quality. The Baidu Encyclopedia has more overall entries than Wikipedia; therefore, it is reasonable for Baidu Encyclopedia to have more entries in the category of liver disease than Wikipedia. In Wikipedia, terms can be easily linked to each other, making it easier for readers to build connections of knowledge [29,30]. In Baidu Encyclopedia, although there are abundant entries, the isolated entries are not linked with the other related entries, thereby making it unconducive to the formation of knowledge networks for some concepts.

We used the DISCERN instrument to evaluate the quality and reliability of the articles in Baidu Encyclopedia and Wikipedia. The DISCERN scores of Wikipedia entries were significantly higher than those of Baidu Encyclopedia entries. Consistent with that reported in previous studies, Wikipedia is a good resource of health information on the internet $[8,14]$. In section 1 of the DISCERN instrument, which reflects the reliability of articles, the scores for the entries in Baidu Encyclopedia were significantly lower than those for the articles in Wikipedia, indicating that the entries in Baidu Encyclopedia were less reliable than those in Wikipedia. We observed that most of the Baidu Encyclopedia entries lacked reference sources, while Wikipedia includes a detailed source for almost every entry. A high-quality citation source is an essential guarantee for the reliability of a paper [31]. However, several studies have found that Wikipedia is difficult for the general public to read and is not a reliable source for medical students [25,32]. During the 2019 follow-up, we observed the source of citations for each paper. The sources of Baidu Encyclopedia references were found to be ambiguous; they were mostly search results of Baidu academic search (an academic search engine of Baidu) and did not represent the literature cited by the entry. However, citations in Wikipedia can be explicitly identified and have original links to academic databases such as PubMed. Accurate referencing is crucial for improving the authoritativeness and credibility of a paper $[31,33]$. In this regard, Wikipedia was found to be better than Baidu Encyclopedia, as the basic ideas are cited and most of the references are academic papers, which guarantee scientific accuracy and reliability. In section 2 of the DISCERN instrument, which assesses the quality of the information on treatment choices, the average score of the articles in Wikipedia was found to be higher than that of the entries in Baidu Encyclopedia. However, the difference between them was not statistically significant. The scores of both Wikipedia and Baidu Encyclopedia entries were low in section 2. The quality of the information on treatment choices in both Wikipedia and Baidu Encyclopedia was not satisfactory, which is in accordance with that reported in previous studies [16,25,32]. After all, Wikipedia and Baidu Encyclopedia are not professional medical websites. Both Wikipedia and Baidu Encyclopedia have room for further 
improvement in providing information on treatment. Since the choice of treatment is an important target for patients to search for, adequate information should also be provided for the choice of treatment. References to international guidelines for various diseases and attracting, encouraging, and even recruiting more medical professionals to participate in the editing of the medical information provided in these websites can improve the quality of the information provided in these websites. Further, patients should be advised to seek treatment advice from more professional medical websites, and it would be more reliable to seek professional advice directly from medical personnel [34]. In section 3 of the DISCERN instrument, the score for Baidu Encyclopedia entries was lower than that for Wikipedia articles, which is consistent with the evaluation result of the total score.

In terms of the update timeliness, Wikipedia was significantly better than Baidu Encyclopedia in 2016 and 2019. During the 3-year follow-up, all Wikipedia pages were updated on time. As for Baidu Encyclopedia, among the 25 entries, 11 entries were not updated in 3 years and the longest update time reached 2564 days. Wikipedia is updated regularly and it evolves continuously while the content in Baidu Encyclopedia has been rarely maintained since its generation. The low update frequency has caused some information to be outdated, and some outdated information may even be wrong. Therefore, the timeliness of information is also an important aspect influencing the quality of medical information.

Our study uses the DISCERN instrument to evaluate the quality of Baidu Encyclopedia entries and compares the quality of these entries with that of Wikipedia articles. This is the first study to assess the quality of health information in Baidu Encyclopedia with the DISCERN instrument. Moreover, this is the first study to follow up on the quality of encyclopedia websites for 3 years. In 3 years, we found that the quality of the articles of both websites had not improved significantly.

\section{Limitations}

Our study design does have some limitations. First, the results of our research only reflect the information on liver diseases in Baidu Encyclopedia and Wikipedia on September 20, 2016, and September 20, 2019. Second, the DISCERN instrument is the only tool used to evaluate article quality in this study. Although the DISCERN instrument is widely used to evaluate website information, comprehensive use of more evaluation tools may make the results more convincible. The LIDA tool is a web-based validation instrument to measure the design and content of health information on the internet [35]. We plan to use the LIDA tool to evaluate the quality of articles in Baidu Encyclopedia and Wikipedia in our future research.

\section{Conclusion}

Although Wikipedia is not as good as the Baidu Encyclopedia in terms of the number of entries, its reliability is better than that of Baidu Encyclopedia, thereby making it more helpful to web-based health information seekers. However, the quality of the treatment choices in both Wikipedia and Baidu Encyclopedia's entries is not satisfactory. Wikipedia is updated frequently to keep the information up-to-date. The entries in Baidu Encyclopedia lack maintenance and are not updated on time; some information is outdated and some new content are lacking. In order to find accurate health information, people are advised to seek the help of medical professionals instead of looking for a prescription amid the confusing information on the internet.

\section{Acknowledgments}

Fei Sun would like to thank his wife, Wenchao Kong, for her patience, understanding, and great support during this work.

\section{Conflicts of Interest}

None declared.

\section{Multimedia Appendix 1}

International Statistical Classification of Diseases and Related Health Problems 10th Revision code search terms for liver diseases in Baidu Encyclopedia in 2019.

[PDF File (Adobe PDF File), 110 KB-Multimedia Appendix 1]

\section{Multimedia Appendix 2}

International Statistical Classification of Diseases and Related Health Problems 10th Revision code search terms for liver diseases in Wikipedia in 2019.

[PDF File (Adobe PDF File), 29 KB-Multimedia Appendix 2]

\section{Multimedia Appendix 3}

The DISCERN instrument.

[DOC File, 33 KB-Multimedia Appendix 3]

\section{References}

1. Internet World Stats. World Internet Users and 2019 Population Stats. URL: http://www.internetworldstats.com/stats.htm [accessed 2019-06-30] 
2. CNNIC. China Internet Network Development State Statistic Report. URL: http://www.cac.gov.cn/2019-08/30/c_1124939590. $\underline{\mathrm{htm}}$ [accessed 2019-08-30]

3. Jin J, Yan X, Li Y, Li Y. Int J Med Inform 2016 Feb;86:91-103. [doi: 10.1016/j.ijmedinf.2015.11.002] [Medline: 26616406]

4. Zhao Y, Zhang J. Consumer health information seeking in social media: a literature review. Health Info Libr J 2017 Dec;34(4):268-283 [FREE Full text] [doi: 10.1111/hir.12192] [Medline: 29045011]

5. Bao H, Zhu F, Wang F, Liu Z, Bao MH, He S, et al. Scoliosis related information on the internet in China: can patients benefit from this information? PLoS One 2015;10(2):e0118289 [FREE Full text] [doi: 10.1371/journal.pone.0118289] [Medline: 25689064]

6. Jo JH, Kim JR, Kim MJ, Chung JW, Park JW. Quality and readability of online information on dental treatment for snoring and obstructive sleep apnea. Int J Med Inform 2020 Jan;133:104000. [doi: 10.1016/j.ijmedinf.2019.104000] [Medline: 31731221]

7. Baumann E, Czerwinski F, Reifegerste D. Gender-Specific Determinants and Patterns of Online Health Information Seeking: Results From a Representative German Health Survey. J Med Internet Res 2017 Apr 04;19(4):e92 [FREE Full text] [doi: 10.2196/jmir.6668] [Medline: 28377367]

8. Laurent MR, Vickers TJ. Seeking health information online: does Wikipedia matter? J Am Med Inform Assoc 2009;16(4):471-479 [FREE Full text] [doi: 10.1197/jamia.M3059] [Medline: 19390105]

9. Wikipedia. Wikipedia. URL: https://www.wikipedia.org/ [accessed 2019-09-15]

10. Farič N, Potts HWW. Motivations for contributing to health-related articles on Wikipedia: an interview study. J Med Internet Res 2014 Dec 03;16(12):e260 [FREE Full text] [doi: 10.2196/jmir.3569] [Medline: 25498308]

11. Thomas GR, Eng L, de Wolff JF, Grover SC. An evaluation of Wikipedia as a resource for patient education in nephrology. Semin Dial 2013;26(2):159-163. [doi: 10.1111/sdi.12059] [Medline: 23432369]

12. Hasty RT, Garbalosa RC, Barbato VA, Valdes PJ, Powers DW, Hernandez E, et al. Wikipedia vs peer-reviewed medical literature for information about the 10 most costly medical conditions. J Am Osteopath Assoc 2014 May;114(5):368-373. [doi: 10.7556/jaoa.2014.035] [Medline: 24778001]

13. Baidu. Baidu Encyclopedia. URL: http://baike.baidu.com/ [accessed 2019-09-15]

14. Weiner SS, Horbacewicz J, Rasberry L, Bensinger-Brody Y. Improving the Quality of Consumer Health Information on Wikipedia: Case Series. J Med Internet Res 2019 Mar 18;21(3):e12450 [FREE Full text] [doi: 10.2196/12450] [Medline: 30882357]

15. Yacob M, Lotfi S, Tang S, Jetty P. Wikipedia in Vascular Surgery Medical Education: Comparative Study. JMIR Med Educ 2020 Jun 19;6(1):e18076 [FREE Full text] [doi: 10.2196/18076] [Medline: 32417754]

16. Modiri O, Guha D, Alotaibi NM, Ibrahim GM, Lipsman N, Fallah A. Readability and quality of wikipedia pages on neurosurgical topics. Clin Neurol Neurosurg 2018 Mar;166:66-70. [doi: 10.1016/j.clineuro.2018.01.021] [Medline: 29408776]

17. Beijing Morning Post. Rainbow Plan. 2012 Dec 10. URL: http://tech.qq.com/a/20121210/000054.htm [accessed 2019-06-30]

18. Wong MCS, Huang JLW, George J, Huang J, Leung C, Eslam M, et al. The changing epidemiology of liver diseases in the Asia-Pacific region. Nat Rev Gastroenterol Hepatol 2019 Jan;16(1):57-73. [doi: 10.1038/s41575-018-0055-0] [Medline: 30158570]

19. Na X, Guo H, Zhang Y, Shen L, Wu S, Li J. Mining Open Payments Data: Analysis of Industry Payments to Thoracic Surgeons From 2014-2016. J Med Internet Res 2018 Nov 30;20(11):e11655 [FREE Full text] [doi: 10.2196/11655] [Medline: $\underline{30504119]}$

20. Musy SN, Endrich O, Leichtle AB, Griffiths P, Nakas CT, Simon M. Longitudinal Study of the Variation in Patient Turnover and Patient-to-Nurse Ratio: Descriptive Analysis of a Swiss University Hospital. J Med Internet Res 2020 Apr 02;22(4):e15554 [FREE Full text] [doi: 10.2196/15554] [Medline: 32238331]

21. Jabaley CS, Groff RF, Barnes TJ, Caridi-Scheible ME, Blum JM, O'Reilly-Shah VN. Sepsis information-seeking behaviors via Wikipedia between 2015 and 2018: A mixed methods retrospective observational study. PLoS One 2019;14(8):e0221596 [FREE Full text] [doi: 10.1371/journal.pone.0221596] [Medline: 31437248]

22. WHO. ICD-10 version: 2016. 2016. URL: http://apps.who.int/classifications/icd10/browse/2016/en [accessed 2016-09-30]

23. Charnock D, Shepperd S, Needham G, Gann R. DISCERN: an instrument for judging the quality of written consumer health information on treatment choices. J Epidemiol Community Health 1999 Feb;53(2):105-111. [doi: 10.1136/jech.53.2.105] [Medline: 10396471$]$

24. Azer SA, AlSwaidan NM, Alshwairikh LA, AlShammari JM. Accuracy and readability of cardiovascular entries on Wikipedia: are they reliable learning resources for medical students? BMJ Open 2015 Oct 06;5(10):e008187 [FREE Full text] [doi: 10.1136/bmjopen-2015-008187] [Medline: 26443650]

25. Azer SA. Is Wikipedia a reliable learning resource for medical students? Evaluating respiratory topics. Adv Physiol Educ 2015 Mar;39(1):5-14 [FREE Full text] [doi: 10.1152/advan.00110.2014] [Medline: 25727464]

26. Prasanth AS, Jayarajah U, Mohanappirian R, Seneviratne SA. Assessment of the quality of patient-oriented information over internet on testicular cancer. BMC Cancer 2018 May 02;18(1):491 [FREE Full text] [doi: 10.1186/s12885-018-4436-0] [Medline: 29716564]

27. Azer SA, Alghofaili MM, Alsultan RM, Alrumaih NS. Accuracy and Readability of Websites on Kidney and Bladder Cancers. J Cancer Educ 2018 Aug;33(4):926-944. [doi: 10.1007/s13187-017-1181-z] [Medline: 28281091] 
28. Zhu X, Qiu X, Wu D, Chen S, Xiong J, Du H, et al. Reliability of information about the use of antiepileptic drugs during pregnancy from three major web search engines in China. PLoS One 2018;13(12):e0208783 [FREE Full text] [doi: 10.1371/journal.pone.0208783] [Medline: 30586373]

29. Good BM, Clarke EL, Loguercio S, Su AI. Building a biomedical semantic network in Wikipedia with Semantic Wiki Links. Database (Oxford) 2012;2012:bar060 [FREE Full text] [doi: 10.1093/database/bar060] [Medline: 22434829]

30. Lamprecht D, Lerman K, Helic D, Strohmaier M. How the structure of Wikipedia articles influences user navigation. New Rev Hypermedia Multimed 2017 Jan 02;23(1):29-50 [FREE Full text] [doi: 10.1080/13614568.2016.1179798] [Medline: 28670171]

31. Heilman JM, West AG. Wikipedia and medicine: quantifying readership, editors, and the significance of natural language. J Med Internet Res 2015 Mar 04;17(3):e62 [FREE Full text] [doi: 10.2196/jmir.4069] [Medline: 25739399]

32. Azer SA. Evaluation of gastroenterology and hepatology articles on Wikipedia: are they suitable as learning resources for medical students? Eur J Gastroenterol Hepatol 2014 Feb;26(2):155-163. [doi: 10.1097/MEG.0000000000000003] [Medline: 24276492]

33. Luyt B, Tan D. Improving Wikipedia's credibility: References and citations in a sample of history articles. J. Am. Soc. Inf. Sci 2010;61(4):715-722. [doi: 10.1002/asi.21304]

34. Biggs TC, Jayakody N, Best K, King EV. Quality of online otolaryngology health information. J Laryngol Otol 2018 Jun;132(6):560-563. [doi: 10.1017/S0022215118000774] [Medline: 30019668]

35. Minervation. Minervation validation instrument for health care web sites, version 1.2.: Minervation; 2011. URL: http:/ /www.minervation.com/wp-content/uploads/2011/04/Minervation-LIDA-instrument-v1-2.pdf [accessed 2019-09-30]

\section{Abbreviations}

ICD-10: International Statistical Classification of Diseases and Related Health Problems 10th Revision

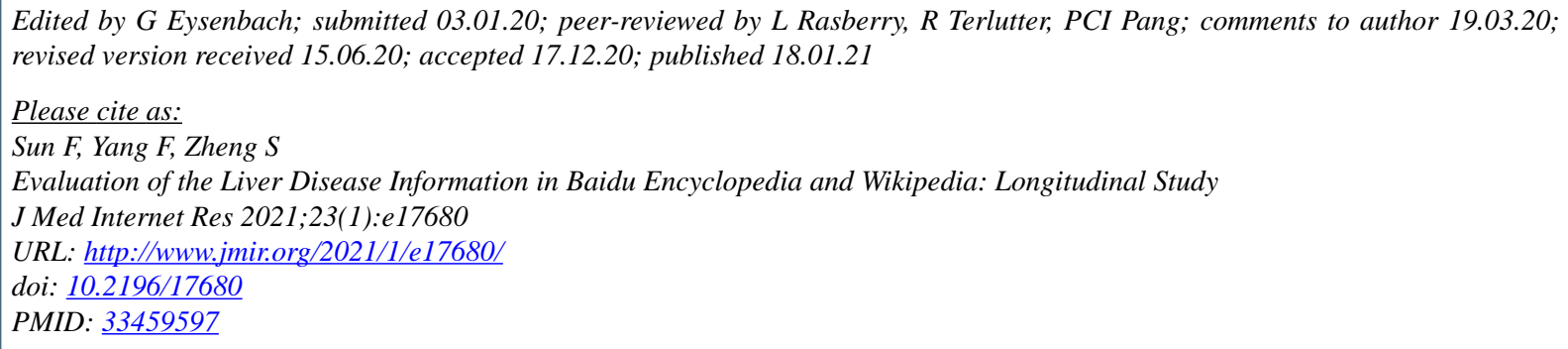

CFei Sun, Fuchun Yang, Shusen Zheng. Originally published in the Journal of Medical Internet Research (http://www.jmir.org), 18.01.2021. This is an open-access article distributed under the terms of the Creative Commons Attribution License (https://creativecommons.org/licenses/by/4.0/), which permits unrestricted use, distribution, and reproduction in any medium, provided the original work, first published in the Journal of Medical Internet Research, is properly cited. The complete bibliographic information, a link to the original publication on http://www.jmir.org/, as well as this copyright and license information must be included. 